\title{
Remoción de matorrales semi-áridos en Magallanes: efectos sobre la composición, estructura y rasgos funcionales de los ensambles de aves
}

\author{
Removal of semi arid shrublands in Magallanes: \\ effects on composition, structure and functional diversity of bird assemblages
}

\author{
Alejandro Kusch ${ }^{1}$, Osvaldo Vidal ${ }^{2} \&$ Juan Marcos Henríquez ${ }^{2}$
}

\begin{abstract}
Resumen
La remoción mecánica de matorrales, es una práctica agropecuaria reciente en la estepa semi-árida de Magallanes, que consiste en la remoción parcial de franjas de matorrales para mejorar el acceso del ganado e incrementar la producción de biomasa forrajera. Aunque esta práctica afecta miles de hectáreas, se sabe muy poco sobre los efectos ecológicos asociados que determinan cambios en la vegetación y la fauna dependiente. Investigamos el efecto integrado del control mecánico en la composición, estructura y diversidad funcional de aves del matorral dominado por Romerillo (Chilliotrichum diffusum [G. Fort.] Kuntze), mediante la comparación entre diferentes intensidades de remoción: a) sin remoción, b) remoción intermedia, c) remoción alta. Se evaluó la diversidad de plantas mediante puntos de intercepto, así como la abundancia de aves con parcelas de radio fijo. La información fue analizada mediante procedimientos univariados (KruskalWallis) y multivariados (e.g. MRPP, Indval, RLQ, Fourth-Corner), para relacionar la estructura del hábitat con la composición, estructura y rasgos funcionales de los ensambles de aves. La abundancia y riqueza de aves disminuyeron significativamente cuando la remoción fue alta, no así con remoción intermedia. Se encontraron respuestas especieespecíficas asociadas a los tratamientos, indicando pérdida de identidad del ensamble de aves en ambas situaciones con remoción. Cuatro especies de aves fueron detectadas como indicadoras del matorral sin remoción (Canastero del sur, Cometocino patagónico, Chercán, Zorzal),
\end{abstract}

mientras que sólo una especie fue asociada a los matorrales removidos (Bailarín chico). Las aves insectívoras, migrantes Neotropicales y nidificantes de follaje estuvieron positivamente asociadas a la cobertura del Romerillo, mientras que los omnívoros, nidificantes del suelo, forrajeras del suelo y residentes se asociaron positivamente con la cobertura de graminoides, donde el Romerillo se removió. El matorral parece proporcionar estructuras claves para la asociación positiva con rasgos funcionales y especialistas dependientes de arbustos, a diferencia de los sitios con control mecánico, donde otros especialistas de ambientes abiertos, tienen mayores abundancias.

\section{Palabras clave:}

Rasgos funcionales de las aves, procedimientos multivariados, disturbio de hábitat, Chilliotrichum diffusum, Asthenes anthoides, Anthus correndera

\section{Abstract}

Mechanical removal of shrublands is a recent management practice adopted in the semi-arid steppes of Magallanes. This involves partially removing the shrub cover in strips, in order to improve the access of sheep and facilitate the

\footnotetext{
1 Wildlife Conservation Society - Chile. Balmaceda 586,

Punta Arenas. $\longrightarrow$ akusch@wcs.org

2 Laboratorio de Botánica, Instituto de la Patagonia, Universidad de Magallanes. Punta Arenas, Chile.
} 
production of forage biomass. Although this procedure affects thousands of hectares, very little is known about the ecological effects that could determine changes in the vegetation and associated fauna. We studied the integrated effect of the motorized control of Romerillo (Chilliotrichum diffusum [G. Fort.] Kuntze) shrubland on composition, structure and functional diversity, by comparing different removal intensities: a) without shrub removal, b) intermediate removal, c) high removal of shrub coverage. We evaluated plant diversity by means of intercept-point transects, and bird abundance using point station census. The information was analyzed with univariate (KruskalWallis), and multivariate procedures (e.g. MRPP, Indval, RLQ, Fourth-Corner), in order to correlate habitat structure with the composition and functional traits of bird assemblages. Bird abundance and species richness decreased significantly when shrub removal was high, but not in the case of intermediate removal. We found species-specific responses, associated with all treatments, and loss of bird assemblage identity, after shrub removal. Four species were detected as indicators of natural shrublands (Austral Canastero, Patagonian Sierra Finch, House Wren, Austral Thrush), while only one was associated with removal (Correndera Pipit). Insectivore birds, Neotropical migrants and foliage nesting birds were positively associated with Romerillo coverage, while omnivores, ground nesting, ground feeders and resident birds were associated with graminoids plants when shrubs were removed. Romerillo shrubs seem to provide a key structure for a positive association with the functional traits of specialist birds, in contrast with removed shrub sites, where abundance of other open land specialists were greater.

\section{Key words:}

Functional traits, multivariate procedures, habitat disturbance, Chilliotrichum diffusum, Asthenes anthoides, Anthus correndera

\section{INTRODUCCIÓN}

En ecología, la diversidad biológica es interpretada usualmente a través de indicadores numéricos, índices que resumen información contenida en ensambles de especies para un lugar y tiempo definidos (e.g. MacArthur \& MacArthur 1961), en distintas escalas espaciales (Moreno 2001). En Chile, esta aproximación ha sido extensivamente usada para describir comunidades y ensambles de grupos taxonómicos conspicuos como las aves o las plantas vasculares afectadas por diferentes tipos de perturbaciones o gradientes ecológicos (e.g. Rau et al. 1998, Silva-Rodríguez et al. 2008, Muñoz-Pedreros \& Merino 2014). Sin embargo, los índices de diversidad más usados, a menudo pierden información referente a las comunidades y raramente son complementados con elementos integrados sobre composición, estructura y función del hábitat (Noss 1990), o bien, no son lo suficientemente sensibles a cambios moderados (Bradford et al. 1998, Milesi et al. 2002). Una aproximación integrativa, que incluya descriptores de composición, estructura y función de la biodiversidad de un grupo y su hábitat, debe ser incorporada para entender la complejidad de los efectos de los disturbios en el contexto de conservación, actividades productivas y manejo de recursos naturales (Noss 1990, Vandewalle et al. 2010). En este contexto, la medición de la diversidad funcional es una solución para describir o predecir los cambios en el ambiente, que pudiesen tener un origen antrópico (e. g. Chapin et al. 2000, Flynn et al. 2009). Existen varias aproximaciones metodológicas para su estudio, pero recientemente, el estudio de los rasgos funcionales (Petchet \& Gaston 2006) se ha incrementado notablemente debido al reciente desarrollo de técnicas multivariadas que permiten análisis integrados a nivel comunitario (Kleyer et al. 2012). Los rasgos funcionales de las especies son atributos del fenotipo de un individuo asociados con su adecuación biológica y función ecológica (Violle et al. 2007), que influencia procesos en el ecosistema (Petchet \& Gaston 2006). Usualmente en vertebrados se utilizan rasgos funcionales que identifican la dieta principal, masa corporal o movimientos migratorios, $y$ en aves en particular se utilizan rasgos más específicos como el lugar de forrajeo o el lugar donde se construye un nido. La clave para obtener resultados satisfactorios es utilizar rasgos funcionales lo más específicos posible para el hábitat en el que se están estudiando (Scherer et al. 2016).

Los ensambles de aves representan uno de 
los grupos más utilizados para evaluar disturbios en el ambiente, sean éstos originados por causas naturales o antrópicas (e.g. Wiens 1989, Ralph et al. 1995). Este grupo es relativamente conspicuo $y$ constituye un indicador eficaz para determinar efectos relativos a perturbaciones debido a entre otros, al carácter protector y/o función ecológica de algunas especies claves (e.g. Jaksic et al. 2002, Cornelius et al. 2008). Mas aún, la interacción con el hábitat de éste grupo requiere de un análisis más exhaustivo, porque la relación entre diversidad de aves y la estructura del hábitat puede ser directa, incluso a diferentes escalas (MacArthur \& MacArthur 1961, Ralph 1985). A medida que la escala espacial disminuye, las comunidades de aves se relacionan más estrechamente con la composición florística, y se hace menos relevante la estructura de la vegetación (Knopf et al. 1990, Fleishman et al. 2003). Así, la composición, diversidad florística y formas de vida de las plantas, entre otros atributos, entregan una mejor predicción de los recursos de refugio y alimento disponibles para las aves. De esta manera, la plasticidad en la historia de vida de las aves puede verse restringida si existen alteraciones críticas en el hábitat (Wiens 1989). En casos extremos donde se altera la estructura del tipo vegetacional dominante, incluso a pequeña o mediana escala, consecuentemente se deben esperar resultados en cascada que pueden verse reflejados en los componentes faunísticos (Wiens 1989, Rodewald \& Vitz 2005) y que conducen al recambio de especies y disminución de especialistas (Cid \& Caviedes-Vidal 2014, Ibarra \& Martin 2015).

La avifauna de la Patagonia Chilena-Austral (al sur de los $52^{\circ}$ de latitud, sensu Bannister et al. 2011), se compone de aproximadamente 128 especies de aves terrestres, siendo las principales características de las comunidades el alto porcentaje de aves migrantes neotropicales $y$ el relativamente alto grado de endemismo (Vuilleumier 1998). Sin embargo, en los matorrales semiáridos que representan la transición bosqueestepa Patagónica, el número de especies sería considerablemente menor, dada la distribución más restringida de este tipo de vegetación en Chile Austral y por la simplificación florística y estructural de la vegetación (Pisano 1977). Aunque la avifauna ha sido bien documentada por extensos estudios biogeográficos y sistemáticos (e. g. Vuilleumier 1991, 1995), los estudios ecológicos son más bien escasos, faltando por conocer aún muchas de las relaciones específicas (o generalistas) de las aves con sus hábitats. Más aún, frente a problemas inminentes como el cambio climático, la industrialización creciente y sobreexplotación de recursos, se hace más urgente evaluar los efectos de estas alteraciones sobre los ensambles comunitarios de aves.

En particular, la Región de Magallanes ha estado históricamente sometida a usos agropecuarios extensivos, con miles de hectáreas afectadas por sobrepastoreo, compactación del suelo e incendios. Más recientemente, una de las perturbaciones relacionadas al uso ganadero que acontece en ecosistemas semiáridos australes, es la remoción mecánica de matorrales (llamado localmente corte en franja). Esta remoción se hace para aumentar la superficie de forrajeo y la biomasa disponible para el ganado. Este uso de suelo tiene un potencial impacto en la biodiversidad, pero sus efectos no han sido mayormente evaluados (Blanco et al. 2005, Kusch \& Henríquez 2014). Prácticas similares han sido utilizadas en grandes extensiones de territorio en Norteamérica (Baker et al. 1976) y la pérdida de hábitat, la disminución poblacional de aves especialistas y el recambio de especies está bien documentada (Wiens \& Rotenberry 1985, Lazo et al. 1990, Rodewald \& Vitz 2005). En el hemisferio austral, el control mecánico de matorral ha demostrado disminuir la competencia entre especies leñosas y se logra aumentar la productividad forrajera (Adema et al. 2004). Sin embargo, se ha desatendido en un sentido más amplio los efectos potenciales sobre la fauna asociada. Si bien Kusch \& Henríquez (2014), describieron las diferencias entre ensambles de aves en matorrales con distintos grados de intervención, también surgieron interrogantes sobre las causales de dichas diferencias en la diversidad de los ensambles y abundancias de cada especie. En este sentido, complementamos información previamente publicada, incluyendo atributos funcionales de las aves (i. e. gremios), los que explican las asociaciones de la historia de vida (Ehlers et al. 2016) de las especies de aves en relación a los atributos estructurales del hábitat. Esta aproximación y re-análisis de datos es novedosa al 
menos para los estudios de la avifauna patagónica existentes, y más aún para las descripciones de los impactos de las actividades antropogénicas en el hemisferio sur.

\section{MATERIALES Y MÉTODOS}

Descripción del área de estudio

El área de estudio se encuentra en la parte continental de la Región de Magallanes, Patagonia Chilena, en la localidad de Laguna Blanca (52 $47^{\circ}$ $\left.\mathrm{S}-71^{\circ} 01^{\prime} \mathrm{W}\right)$. Este tipo de ecosistemas ha sido históricamente utilizado por la ganadería (Martinic 1985). El clima es semiárido con precipitación media anual de $300 \mathrm{~mm}$ y vientos dominantes del WNW (Endlicher \& Santana 1988, Butorovic 2006). El tipo de vegetación dominante corresponde a un denso matorral estepario, con dominancia del arbusto Romerillo (Chiliotrichum diffusum), en el ecotono bosque-estepa Patagónica (Moore 1983). La cobertura del matorral en el área es contínua y tiene una cobertura cercana al $50 \%$, ocupando miles de hectáreas. En sectores de colinas y con mayor escorrentía, el matorral es intercalado por zonas menos densas de vegetación y con mayor humedad, denominadas vegas (Pisano 1977). Otras especies asociadas codominantes del matorral son el coirón Festuca gracillima Hook. f., Leptinella scariosa (Cass.) Franch, Deschampsia flexuosa (L.) Trin. y Azorella trifurcata (Gaertn.) Pers. (Pisano 1977). Más información sobre la estructura de la vegetación del área de estudio en Kusch \& Henríquez (2014).

Tratamientos de control mecánico del matorral de Romerillo

Se seleccionaron tres potreros adyacentes y con diferentes manejos del matorral de Romerillo. Estos potreros constituyen la base para una estratificación según uso de suelo, relativo a las intensidades del tratamiento: 1) El primer potrero (sin remoción) consistió en un predio de 270 hectáreas, en el que no existió control mecánico del Romerillo, y cuya cobertura promedio fue cercana al 50\%; 2) El segundo potrero (remoción intermedia), de 300 hectáreas, se dejaron franjas de Romerillo de $8 \mathrm{~m}$, intercaladas con franjas de 10 m en donde se extrajo mecánicamente el matorral; 3) El tercer potrero (remoción alta), consistió en un predio de 300 hectáreas, en el que se mantuvieron franjas de Romerillo de 1 a 4 metros de ancho (Kusch \& Henríquez 2014). La remoción mecánica del Romerillo en los predios 2 y 3 se realizó en el año 2005, un año antes del registro de datos aquí presentado.

\section{Conteo de aves en el matorral de Romerillo}

Los muestreos se realizaron durante el periodo estival entre noviembre y febrero de las temporadas 2006/07 y 2007/08. Los conteos se efectuaron entre las 5:30 y 10:30 de la mañana debido a que la frecuencia y magnitud del viento es menor (ca. 5 m/s, Endlicher \& Santana 1988, Butorovic 2006). En cada tratamiento se dispuso de una parcela de muestreo rectangular de 25 há, cada una con cuatro estaciones de escucha de 100 metros de radio. Según los análisis de duración de conteo y radio de cada estación en ambientes abiertos (Saavard \& Hooper 1997), cada estación de escucha se ubicó a 100 metros de distancia de la siguiente estación y la duración de los conteos fue de $10 \mathrm{~min}$. La parcela se localizó en su punto de partida al azar, siguiendo una dirección que mantuviera un máximo de distancia respecto del borde del hábitat y evitando inclusiones de parches de otras comunidades de vegetación como vegas. En los conteos sólo se registraron individuos que hicieron uso del hábitat (e. g. alimentación, canto, nidificación). En aquellos individuos que manifestaron comportamiento territorial o reproductivo mediante cantos repetitivos y/o vuelo estacionario se les contabilizó sólo una vez para no sobreestimar su abundancia. En total se realizaron 20 conteos para cada potrero (60 en total, 10 horas sumadas de registro), los que debido a su independencia espacial fueron considerados como unidades de muestreo para los análisis.

Registro de la composición florística y estructura del matorral de Romerillo

Se registró la riqueza de plantas vasculares en cada uno de los potreros antes descritos, estimando la composición y cobertura relativa de las plantas usando la técnica de punto intercepto (Mueller- 
Dombois \& Ellenberg 1974). Para cada estación de escucha de aves, se dispusieron transectos de 100 $\mathrm{m}$ de longitud, y en cada transecto se sortearon 5 puntos aleatoriamente a partir de los cuales se extendieron líneas perpendiculares de $5 \mathrm{~m}$ de largo. Para cada línea se registró la planta que interceptó un punto cada $5 \mathrm{~cm}$ (100 puntos en total). Los puntos de intersección para cada transecto fueron transformados a valores de cobertura relativa para cada especie registrada. Además, las especies fueron clasificadas según la forma de vida general (herbáceas, arbustivas, graminoides), para caracterizar atributos sobre estructura de la vegetación de acuerdo a las coberturas estimadas. La determinación taxonómica de las especies se efectuó mediante literatura ad. hoc (e. g. Moore 1983, Matthei 1995, Marticorena 2006), y fue comparada con ejemplares de herbario del Instituto de la Patagonia de la Universidad de Magallanes (HIP, Punta Arenas). La nomenclatura siguió a Henríquez et al. 1995 y fue actualizada siguiendo los registros del sistema de información botánica "Tropicos" del "Missouri Botanical Garden".

\section{Análisis de datos}

Las diferencias sobre la abundancia y composición de especies de aves dentro de los grupos afectados por diferentes intensidades control mecánico de Romerillo (tratamientos), fueron evaluadas mediante dos aproximaciones. La primera de ellas se realizó mediante comparaciones entre grupos usando la prueba univariada no paramétrica Kruskall-Wallis (Motulsky 2007), para las métricas número total de individuos, riqueza y abundancia de cada especie. Este análisis fue complementado, con un procedimiento multivariado denominado "Procedimiento de Multi-Respuestas Permutadas" (en inglés, "MultiResponse Permutation Procedure" [MRPP]) (McCune \& Grace 2002), usando la distancia euclidiana de la abundancia absoluta de los individuos registrados. MRPP es un procedimiento no paramétrico que proporciona un estadístico " $T$ " que describe la separación entre los tratamientos (o grupos), con métricas mostrando la fuerza de la separación. Otro estadístico "A", describe la homogeneidad dentro de grupos en función a lo esperado por azar (denominado tamaño del efecto)
(McCune \& Grace 2002).

Para evaluar fidelidad de especies de aves a tratamientos particulares (o conjuntos de ellos), se realizó el "Análisis de Especies Indicadoras" (en inglés, "Indicator Species Analysis", IndVal (Dufrene \& Legendre 1997, de Cácereset al. 2010). Este análisis evalúa la importancia relativa de las especies en grupos particulares de observaciones (censos), definidos por el usuario, mediante la modelación permutada de las frecuencias y las abundancias observadas en los tratamientos.

Para visualizar gradientes y distancias ecológicas entre unidades de muestreo, atributos del hábitat y diversidad funcional de aves en los diferentes tratamientos, se realizó una ordenación multivariada denominada RLQ (Doledec et al. 1996). Este método evalúa la estructura conjunta de tres matrices denominadas " $R$ " (matriz de variables ambientales), " $L$ " (matriz de especies de aves) y matriz "Q" (matriz con rasgos funcionales de las aves). Las variables incluidas en la matriz " $R$ " fueron la cobertura arbustiva del Romerillo (rom), la cobertura de graminoides (gra), la cobertura de herbáceas (her), la cobertura de biomasa seca o rastrojo (ras) y la riqueza de especies de plantas (riq). La tabla " $L$ " en tanto, consideró la abundancia absoluta de especies de aves registradas en los conteos. Finalmente la matriz " $Q$ ", consistente en una tabla de contingencia, incluyó los siguientes atributos funcionales de las aves: a) dieta principal (herbívoro, insectívoro, omnívoro); b) localización del forrajeo (aéreo, en el suelo, follaje, facultativo); c) localización de nido (cavidades, follaje, suelo) y; d) migración (residente, migrante). Los rasgos funcionales de las aves fueron determinados mediante literatura de campo (e. g. Ojeda \& Trejo 2002, Couve \& Vidal 2007, McGehee \& Eitniear 2007, Kelt et al. 2012) y conocimiento experto (Tabla 1). Debido a que el análisis RLQ es estrictamente exploratorio, la relación combinada de las matrices fue evaluada mediante el método complementario denominado "Análisis de la Cuarta Esquina" (en inglés, "Fourth Corner Analysis"). Este procedimiento describe la relación entre los rasgos funcionales de las aves y las variables ambientales (un rasgo contra una variable a la vez), mediante el coeficiente de correlación de Pearson que describe la fuerza de la relación (Dray \& Legendre 2008), analizando cada combinación de rasgos y variables ambientales a la vez. La 
Tabla 1. Especies de aves presentes en el área de estudio, incluyendo códigos utilizados para los rasgos funcionales: fuente primaria de alimento (gra= granívoro, fac= facultativo, ins= insectivoro, omn= omnívoro), localización del forrajeo (air = aire, fac = facultativo, fol= follaje, sue = suelo), ubicación de nidos (cav= cavidades, fol= follaje, sue= suelo), tipo de migración (neo=neotropical, res= residente anual).

\begin{tabular}{|c|c|c|c|c|}
\hline Nombre científico & $\begin{array}{c}\text { Fuente primaria } \\
\text { de alimento }\end{array}$ & $\begin{array}{l}\text { Localización del } \\
\text { forrajeo }\end{array}$ & $\begin{array}{l}\text { Ubicación de } \\
\text { los nidos }\end{array}$ & Migración \\
\hline Anthus correndera & ins & sue & sue & neo \\
\hline Asthenes anthoides & ins & sue & fol & res \\
\hline Curaeus curaeus & omn & fac & fol & res \\
\hline Lessonia rufa & ins & sue & sue & neo \\
\hline Phrygilus patagonicus & her & fac & fol & res \\
\hline Sturnella loica & omn & sue & sue & res \\
\hline Tacychineta meyenii & ins & air & cav & neo \\
\hline Troglodytes aedon & ins & fol & fol & res \\
\hline Turdus falklandii & ins & sue & fol & res \\
\hline Zonotrichia capensis & omn & $\mathrm{fac}$ & fac & neo \\
\hline
\end{tabular}

significancia estadística de estas relaciones fue determinada mediante el modelo de permutación 5 , que permuta los valores de las especies y de los sitios (Dray \& Legendre 2008).

La estadística descriptiva univariada se realizó con el programa GraphPad Prism v.5.0 (Motulsky 2007), mientras que los análisis multivariados RLQ, Fouth-Corner Analysis, MRPP e IndVal se realizaron usando los paquetes labdsv (Roberts 2006) y ade4 (Dray \& Legendre 2008) del ambiente de programación RStudio ( $\mathrm{R}_{-}$ Development_Core_Team 2012).

\section{RESULTADOS}

Efecto de la remoción del matorral sobre la riqueza y composición de los ensambles de aves

En las 60 unidades de muestreo de los 3 tratamientos, se registraron 1.130 individuos (mediana 34,5, RIQ [rango inter cuartil] 27,244,7) de 10 especies de aves (mediana 4,0, RIQ 3,0 - 5,0). Los taxa más abundantes fueron Chincol (Zonotrichia capensis) (mediana 14,15, RIQ 5,2-24,7), Bailarín Chico (Anthus correndera) (mediana 7,0, RIQ 2,0-15,7) y Colegial (Lessonia rufa) (mediana 2,0, RIQ 0-5,0). Al considerar los tratamientos por separado, la composición total de especies cambió significativamente. El MRPP mostró que el promedio de las diferencias entre grupos fue mayor (distancia promedio $T=0,5686)$, que el promedio de las diferencias intra grupos (distancia promedio $T=0,4645$ ) $(A=0,1239, \quad p<0,001)$, indicando que el control mecánico de Romerillo tiene un efecto significativo en la composición de los ensambles de aves. Similarmente, la prueba de KruskalWallis mostró una disminución estadísticamente significativa de la riqueza de especies $(K=26,78$, $p<0,001)$ y abundancia de individuos $(K=7,54$, $p=0,0023$ ) entre el tratamiento sin remoción y la remoción alta, pero esta disminución no es significativa si se compara el tratamiento control con el tratamiento de remoción intermedia (Figs. 1a, 1b).

La riqueza acumulada de especies para los sitios sin remoción de Romerillo fue de 10 especies (mediana 5,0, RIQ 4,2-6,0), mientras que la riqueza acumulada de especies para los disturbios intermedio y alto fue de seis (mediana 3,0, RIQ 2,2-4,0), y cinco especies (mediana 3,0, RIQ 3,0-4,0), respectivamente (Fig. 1a). Las especies más abundantes en los sitios sin remoción fueron Chincol (mediana 20, RIQ 14,0-30,5) (Fig. 11), Loica (Sturnella loyca) (mediana 5,0, RIQ 3,0-7,0) (Fig. 1h), y Canastero del Sur (Asthenes anthoides) (mediana 3,5, RIQ 0,2-8,7) (Fig. 1d). Para el tratamiento de remoción intermedia, las especies más abundantes fueron nuevamente Chincol (mediana 20,0, RIQ 5,2-24,5) (Fig. 11), 

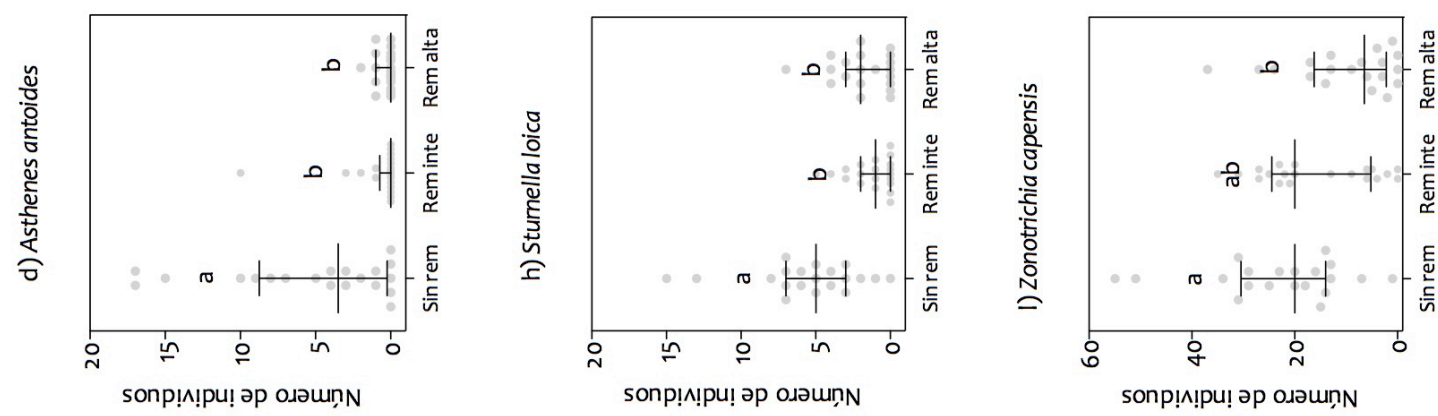

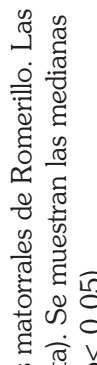

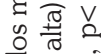
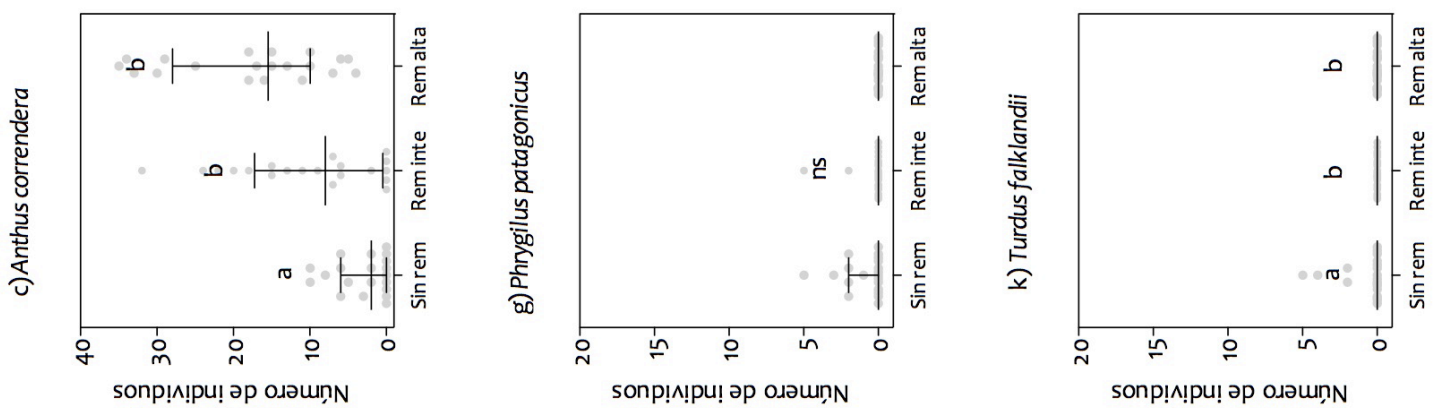

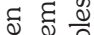

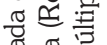

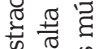

के :0 ?

¿

ช

.

क्ष हี

ชู ผै

ชิ
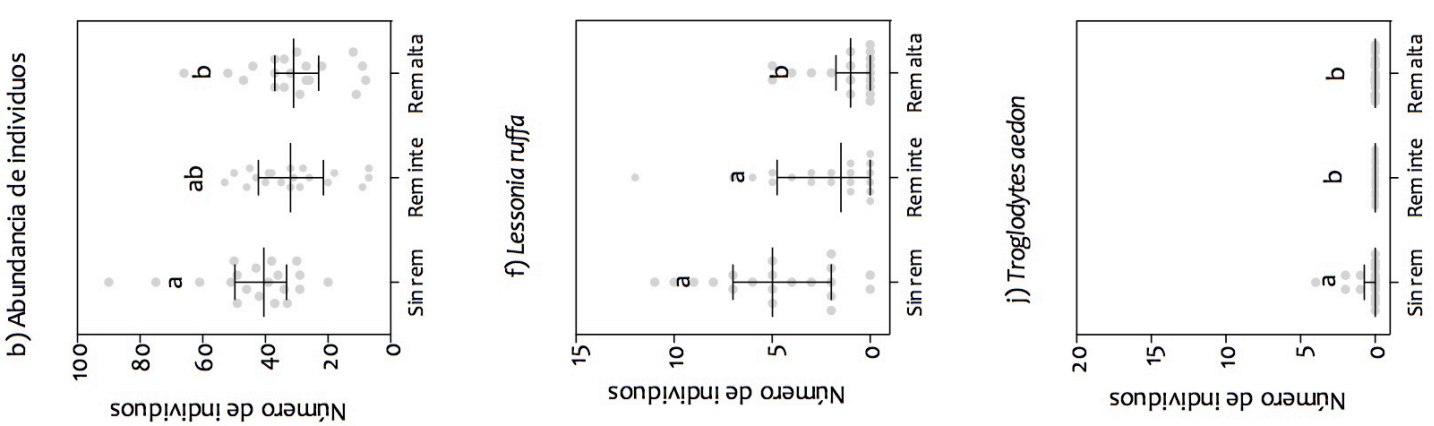

先

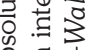

: $\frac{1}{0}$

을 온

눙

ธิ

ते छृ ट्रे

N

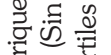

뜨 잉 국

\%

\& है
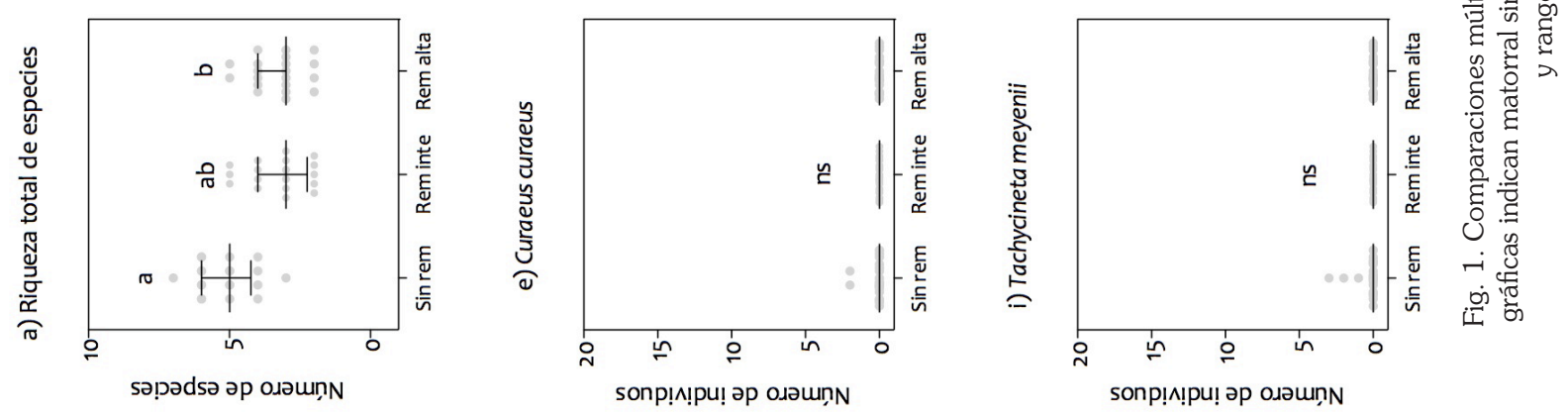
Bailarín Chico (mediana 8,0, RIQ 0,5-17,2) (Fig. 1c) y Colegial (mediana 1,5, RIQ 0-4,7) (Fig. 1f). El matorral con remoción alta del Romerillo mostró que las especies más abundantes fueron Bailarín Chico (mediana 15,5, RIQ 10,0-28,0) (Fig. 1c), Chincol (mediana 6,5, RIQ 2,2-16,2) (Fig. 1l), y Loica (mediana 2,0, RIQ 0-3,0) (Fig. 1h). La comparación múltiple del MRPP mostró que la disimilitud entre el grupo control y el grupo de remoción intermedia fue de $T=0,545$, mientras que entre el grupo de remoción intermedia y alta fue de $T=0,518$. Como era de esperar, la mayor disimilitud se observó entre el grupo sin remoción y de remoción alta, con un valor de $T=0,642$.

El análisis IndVal reveló que 5 especies del ensamble pueden ser consideradas como indicadoras de uno $\mathrm{u}$ otro tratamiento. Cuatro de ellas son especies indicadoras del grupo control sin intervención: Canastero del Sur (Asthenes anthoides, IndVal $=0,782, p<0,001)$, Chercán (Troglodytes aedon, IndVal $=0,500, p=0,013$ ),
Cometocino Patagónico (Phrygilus patagonicus, IndVal $=0,498, \quad p=0,013$ ) y Zorzal (Turdus falklandii, IndVal $=0,447, p=0,025)$. En tanto, el Bailarín Chico (Anthus correndera), resultó ser una especie típica de los matorrales perturbados de intensidad media y alta (IndVal $=0,889, p<$ 0,001).

Respuesta de rasgos funcionales de las aves en matorrales de Romerillo intervenidos

La ordenación multivariada RLQ mostró que existe un claro gradiente en la descomposición de los valores propios ("eigenvalues"), con una covarianza total explicada del $69,19 \%$. El eje principal resumió el $61,89 \%$ de la covarianza, y el eje secundario explicó el 7,30\% de la varianza de la estructura conjunta de las tres matrices (Fig. 2). Los sitios control quedaron claramente separados de los sitios intervenidos en el espacio bi-dimensional, con rasgos funcionales asociados a uno $u$ otro b) RLQ dieta principal

a) RLQ variables ambientales

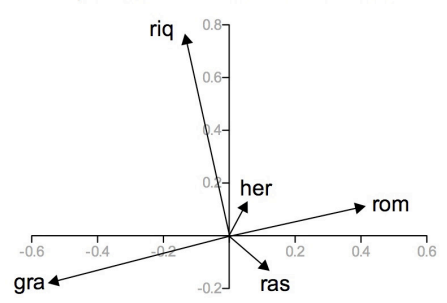

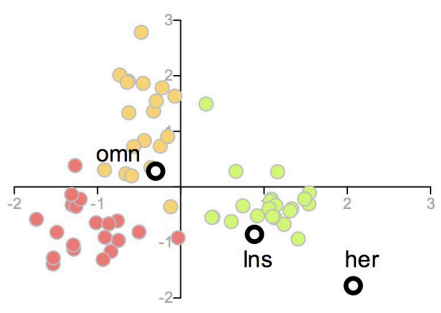

d) RLQ ubicación del nido

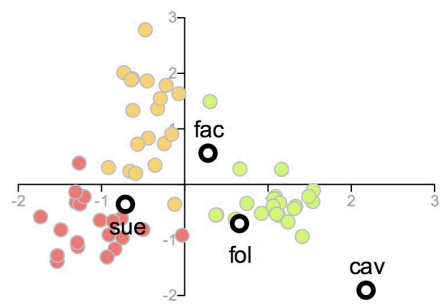

c) RLQ localización del forrajeo

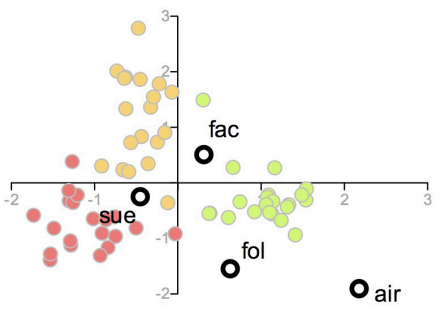

e) RLQ migración

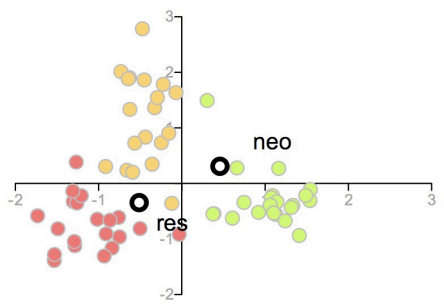

Sin remoción

Remoción intermedia

- Remoción alta

Fig. 2. Ordenación multivariada de tres matrices RLQ mostrando: a) la orientación y fuerza de las variables ambientales en relación a los rasgos funcionales de las aves (her= cobertura de herbáceas, gra= cobertura de gramíneas, ras= cobertura de rastrojo, riq= riqueza de especies de plantas, rom= cobertura de Romerillo), b) orientación de la dieta principal en relación a los tratamientos (her= herbivoros, ins= insectivoros, omn= omnivoros), c) orientación del forrajeo en relación a los sitios de estudio ( $a i r=$ aire, fac= facultativo, fol= follaje, sue= suelo), d) orientación de la localización del nido en relación a los sitios de estudio (cav= cavidades, fac $=$ facultativo, fol= follaje, sue $=$ suelo), y e) orientación del tipo de migración en relación a los sitios de estudio (neo= neotropicales, res= residentes anuales) (covarianza explicada 69,19\%). 
Tabla 2. Resultados del análisis de la cuarta esquina, mostrando el valor de correlación entre las variables ambientales y los rasgos funcionales de las aves. Las correlaciones estadísticamente significativas se muestran en negrita $(p<0,05)$. Variables ambientales: rom= cobertura del romerillo, gra= cobertura de graminoides, her $=$ cobertura de herbáceas, ras= cobertura de rastrojo, riq= riqueza de especies de plantas.

\begin{tabular}{lccccc}
\hline & \multicolumn{3}{c}{ Variables ambientales } \\
\hline $\begin{array}{l}\text { Rasgo funcional } \\
\text { Dieta principal }\end{array}$ & rom & gra & her & ras & riq \\
Herbivoro & & & & & $-0,040$ \\
Insectivoro & 0,023 & $-0,084$ & 0,061 & 0,036 & $\mathbf{- 0 , 1 5 3}$ \\
Omnivoro & $\mathbf{0 , 1 6 8}$ & $\mathbf{- 0 , 1 8 1}$ & $-0,042$ & 0,035 & $\mathbf{0 , 1 5 9}$ \\
& $\mathbf{- 0 , 1 7 1}$ & $\mathbf{0 , 1 9 5}$ & 0,031 & $-0,041$ & $-0,015$ \\
Conducta forrajera & & & & & $-0,107$ \\
Aire & & & & 0,064 & $-0,007$ \\
Follaje & 0,018 & $-0,045$ & 0,007 & 0,037 & $-0,036$ \\
Suelo & 0,034 & $-0,055$ & 0,005 & $-0,044$ & 0,010
\end{tabular}

Ubicación del nido

$\begin{array}{lccccr}\text { Cavidades } & 0,018 & -0,045 & 0,007 & 0,063 & -0,014 \\ \text { Follaje } & \mathbf{0 , 1 6 3} & -0,106 & 0,033 & 0,020 & -0,107 \\ \text { Suelo } & \mathbf{- 0 , 1 4 1} & \mathbf{0 , 2 2 4} & -0,061 & -0,030 & 0,005 \\ \text { Facultativo } & 0,085 & \mathbf{- 0 , 1 2 8} & 0,032 & 0,007 & 0,079\end{array}$

Tipo de migración

$\begin{array}{lrrrrr}\text { Neotropical } & \mathbf{0 , 1 8 9} & \mathbf{- 0 , 2 2 0} & -0,021 & 0,025 & 0,018 \\ \text { Residente anual } & \mathbf{- 0 , 1 8 9} & \mathbf{0 , 2 2 0} & 0,021 & -0,025 & -0,018\end{array}$

tratamiento. El eje principal resultó estar fuerte y positivamente correlacionado con la abundancia del Romerillo (rom, $\sigma=0,868, p<0,001$ ), y fuerte y negativamente correlacionado con la cobertura de gramineas (gra, $\sigma=-0,929, p<0,001$ ) (Fig. 2). Las variables de cobertura herbáceas (her), cobertura de rastrojos (ras) y riqueza de especies (riq) no estuvieron significativamente correlacionadas con el eje principal. Por otra parte, la única variable significativamente correlacionada con el segundo eje fue la riqueza de especies (riq, $\sigma=$ $0,8049, \quad p<0,001)$. Complementariamente, el "análisis de la cuarta esquina" mostró asociaciones significativamente importantes de algunos rasgos funcionales de las aves con los atributos del matorral de Romerillo. Por ejemplo, la cobertura de
Romerillo se asocia significativa y positivamente a las aves insectivoras, pero negativamente a las aves omnivoras y aves que nidifican en el suelo (Tabla 2). Además, el Romerillo afectó positivamente la presencia de aves migrantes Neotropicales, mientras que las aves residentes se asociaron negativamente al Romerillo. La presencia de plantas graminoides estuvo negativamente asociada a las aves insectívoras, y que forrajean en diferentes estratos de vegetación (facultativas), mientras que se detectó asociación positiva con especies de dieta omnívora, con conducta de forrajeo y ubicación de nido en el suelo. Además la cobertura de graminoides se asoció o positivamente con especies residentes anuales, mientras que las especies migratorias se asociaron negativamente 
a esta variable. Finalmente la riqueza de especies de plantas se asoció negativamente con aves de dieta insectívora, mientras que las omnívoras se asociaron positivamente a esta variable.

\section{DISCUSIÓN}

Estudios en ecosistemas de bosques templados y tropicales han demostrado que los rasgos funcionales de ensambles de fauna se correlacionan con la disponibilidad de nichos, alterándose la identidad de la comunidad frente a cambios producidos en la estructura de la vegetación, principalmente por la pérdida de especialistas (e. g. Azeria et al. 2011, Ehlers Smith et al. 2015, Ibarra \& Martin 2015). Por su parte, se ha descrito que los ecosistemas de matorrales semiáridos albergan comunidades aviares con relativamente pocas especies (Wiens 1989), y que la alteración de los parches de hábitat disminuye la diversidad y especies propias de estos ecosistemas con efectos a escala de paisaje (Knick \& Rotenberry 1995). En Patagonia Austral, la avifauna que habita en las asociaciones de matorral de romerillo no son la excepción (Vuilleumier 1998), y los disturbios antropogénicos no sólo provocan una disminución de la riqueza de especies y diversidad (Kusch \& Henríquez 2014), sino que también la intervención sobre la especie vegetal estructuradora desencadenó cambios en la identidad de la comunidad aviar, con consecuencias en los rasgos funcionales de los nuevos componentes del ensamble.

La dependencia de la comunidad aviar de matorrales sin intervención está asociada a la ubicación de los nidos entre el follaje, y a los recursos alimenticios que ofrecen los matorrales. En consecuencia, la remoción del matorral significó la perdida de estos recursos y el recambio de los ensambles de aves. Sirami et al. (2009), encontraron que en matorrales semi-áridos de Namibia el óptimo en riqueza de aves se registró con coberturas entre el 30-60\% del matorral, como las registradas para el matorral de romerillo en áreas sin remoción. Asociaciones entre estos rasgos funcionales y calidad del hábitat se han encontrado en otros ambientes, proporcionando evidencia de la importancia de las estrategias reproductivas y de sobrevivencia, acentuada en especialistas (Vickery et al. 2001, Vander Haegen et al. 2002, Hartel et al. 2014). Esta dependencia de especialistas y especies raras, requiere de mayor atención por contener una funcionalidad única, no observable en otros hábitats (Seymour et al.2015). La remoción intermedia y alta del matorral, estuvo acompañada de la colonización de especialistas de ambientes abiertos como Anthus correndera (Edwards et al. 2013), y la disminución en abundancia de casi todas las otras especies del matorral, lo que genera a su vez la homogenización de ensambles de aves a escala de paisaje (Ibarra \& Martin 2015).

Para una especie en particular, Asthenes anthoides, endémica de Patagonia y que ha sido propuesta como indicadora de los ambientes de matorral (Vuilleumier 1997), verificamos que esta especie es sensible a la remoción de arbustos no sólo por la disminución de su abundancia (Kusch \& Henríquez 2014), sino también debido a su especialización en los recursos que el matorral denso ofrece, y por lo tanto es un buen indicador del estado de los ecosistemas esteparios con matorrales. En matorrales mediterráneos de Chile central también se detectó que otros insectivoros (e. g. Leptasthenura aegithaloides y Asthenes humicola) disminuyen drásticamente ante la perturbación antrópica del hábitat (Lazo et al. 1990). Esta asociación positiva entre insectívoros y abundancia de especies leñosas podría guardar relación con la mayor disponibilidad de humedad y a un efecto protector de los arbustos sobre los insectos (Blaum et al. 2009).

Ejemplos de evaluaciones integradas como las presentadas aquí, son cada vez más comunes en otras latitudes, especialmente para pastizales y matorrales de Norteamérica. Para nuestro conocimiento, este estudio representa una de los primeras aproximaciones que evalúan cambios conjuntos en estructura, composición y función de ensambles de aves en matorrales perturbados de la Patagonia austral. Estos estudios son necesarios especialmente para detectar cambios sutiles en gradientes de perturbación sobre especies raras y especialistas, cuya pérdida puede redundar en degradación funcional (Seymour et al. 2015, Scherer et al. 2016). Coincidimos con otras investigaciones donde la ubicación de los nidos y el tipo de recursos alimenticios son rasgos de las aves importantes de evaluar ante futuros impactos 
de origen antrópico, o para conocer el estado de la biodiversidad en zonas de uso agropecuario, particularmente porque la comunidad aviar posee pocas especies y está dominada por unas pocas aves especialistas.

\section{AGRADECIMIENTOS}

Agradecemos al propietario de estancia Divina Esperanza por facilitarnos el acceso a su predio. El primer autor desea manifestar su agradecimiento a Claudio Vidal por dejar entrever la importancia de las actividades antrópicas sobre la avifauna endémica de la Patagonia. Agradecemos a dos revisores anónimos por incorporar sugerencias que mejoraron sustancialmente este trabajo.

\section{LITERATURA CITADA}

Adema, E. O., Buschiazzo, D., Babinee, F. J., Rucci, T. E., \& Gomez Hermida, V. F. (2004). Mechanical control of shrubs in a semiarid región of Argentina and its effect on soil Water content and grasslands productivity. Agricultural Water Management, 68, 185194.

Azeria, E. T., Ibarzal, J, Hébert, C., Boucher, J., Imbeau, L., \& Savard, J-P. (2011). Differential response of bird functional traits to post-fire salvage logging in a boreal ecosystem. Acta Oecologica, 37, 220-229.

Baker, M. F., Eng, R. L., Gashwiller, J. S., Schroeder, M H., \& Braun, C. E. (1976). Conservation committe report on effects of alteration of sagebrush communities on the associated avifauna. Wilson Bulletin, 88, 165-171.

Bannister, J., Vidal, O. J., Teneb, E.,\& Sandoval, V. (2012).Latitudinal patterns and regionalization of plant diversity along a 4270-km gradient in continental Chile. Austral Ecology, 37, 500-509.

Blanco, L. J., Ferrando, C. A, Biurrun, F. N., Orionte, E. L., Namur, P., Recalde, D. J., \& Berone, G. D. (2005). Vegetation responses to roller chopping and buffergrass seeding in Argentina. Rangeland Ecology \& Management, 58, 219-224.

Blaum, N., Seymour, C., Rossmanith, E.,
Schwager, M., \& Jeltsch, F. (2009). Changes in arthropod diversity along a land use driven gradient of shrub cover in savanna rangelands: identification of suitable indicators. Biodiversity Conservation, 18, 1187-1199.

Bradford, D. F., Franson, S. E., Neale, A. C., Heggem, D. T., Miller, G. R., \& Canterbuty, G. E. (1998). Bird species assemblages as indicators of biological integrity in Great Basin rangeland. Environmental Monitoring and Assessment, 49, 1-22.

Butorovic, N. (2006). Resumen meteorológico año 2004. Estación "Jorge Schythe". Anales Instituto de la Patagonia, 33, 65-71.

Cid, F. D., \& Caviedes-Vidal, E. (2014). Differences in bird assemblages between native natural hábitats and small-scale tree plantations in the semiarid midwest of Argentina. Wilson Journal of Ornithology, 126, 673-685.

Cornelius, C., Cockle, C., Politi, N., Berkunsky, I., Sandoval, L., Ojeda, V., Rivera, L., Hunter, M., \& Martin, K., (2008). Cavity nesting birds in neotropical forests: cavities as a potentially limiting resource. Ornitología Neotropical, 19, 253-268.

Couve, E., \& Vidal, C. (2007).Birds Torres del Paine. Fantástico Sur. Punta Arenas, Chile. Chapin, F. S. III, Zavaleta, E. S., Eviner, V. T., Naylor, R. L., Vitousek, P. M., Reynolds, H. L., Hooper, D. U.,...Díaz, S. (2000). Consequences of changing biodiversity. Nature, 405, 234-242.

De Cáceres, M., Legendre, P., \& Moretti, M. (2010). Improving indicator species analysis by combining groups of sites. Oikos, 119, 1674-1684.

Doledec, S., Chessel, D., ter Braak, C. J. F.,\& Champely, S. (1996). Matching species traits to environmental variables: a new threetable ordination method. Environmental and Ecological Statistics, 3, 143-166.

Dray, S., \& Legendre, P. (2008). Testing species traits-environment relationships: the fourthcorner problem revisited. Ecology, 89, 3400-3412.

Dufrene, M., \& Legendre, P. (1997). Species assemblages and indicator species: the need for a flexible asymmetrical approach. 
Ecological Monographs, 67, 345-366.

Edwards, F. A., Edwards, D. P., Hamer, K. C.,\& Davies, R. G. (2013). Impacts of logging and conservation of rainforest to oil palm on the functional diversity of birds in Sundaland. Ibis, 155, 313-326.

Ehlers Smith, Y. C., Ehlers Smith, D. A., Seymour, C. L., Thébault, E., \& van Venn, F. J. F. (2015). Response of avian diversity to habitat modification can be predicted from life-history traits and ecological attributes. Landscape Ecology, 30, 1225-1239.

Endlicher, W.,\& Santana, A. (1988). El clima al sur de la Patagonia y sus aspectos ecológicos. Un siglo de mediciones climatológicas en Punta Arenas. Anales Instituto de la Patagonia, Serie Cienias Naturales, 18, 57-86.

Fleishman, E., McDonal, N., Mac Nally, R., Murphy, D. D., Walters, J., \& Floyd, T. (2003). Effects of floristic, physiognomy and non-native vegetation on riparian bird communities in a Mojave Desert watershed. Journal of Animal Ecology, 72, 484-490.

Flynn, D. F. B., Gogol-Prokurat, M., Nogeire, T., Molinari, N., Trautman Richers, B., Lin, B. B., Simpson, N., Mayfield, M. M., \& DeClerck, F. (2009). Loss of functional diversity under land use intensification across multiple taxa.Ecology Letters, 12, 22-33.

Hartel, T., Hanspach, J., Abson, D. J., Máthé, O., Moga, C. I., \& Fischer, J. (2014). Bird communities in traditional wood-pastures with changing management in Eastern Europe. Basic and Applied Ecology, 15, 385-395.

Henríquez, J. M., Pisano, E.,\& Marticorena, C. (1995). Catálogo de la flora vascular de Magallanes (XII ${ }^{a}$ Region), Chile. Anales del Instituto de la Patagonia, 23, 5-30.

Ibarra, J. T., \& Martin, K. (2015).Biotic homogenization: Loss of avian functional richness and habitat specialists in disturbed Andean temperate forest. Biological Conservation, 192, 418-427.

Jaksic, F. M., Iriarte, J. A., \& Jiménez, J. E. (2002). The raptors of Torres del Paine National Park, Chile: biodiversity and conservation. Revista Chilena de Historia Natural, 75, 449-461.
Kelt, D., A., Engilis, Jr., A. E., Monárdez, J., Walsh, R., Meserve, P. L., \& Gutiérrez, J. R. (2012). Seasonal and multiannual patterns in avian assemblage structure and composition in northern Chilean thorn-scrub. Condor, 114, 30-43.

Kleyer, M., Dray S., de Bello, F., Lepš, J., Pakeman, R. J., Strauss, B., Thuiller, W., \& Lavorel, S. (2012). Assessing species and community functional responses to environmental gradients: which multivariate method? Journal of Vegetation Science, 23, 805-821.

Knopf, F. L., Sedgwick, J. A., \& Inkley, D. B. (1990). Regional correspondence among shrubsteppe bird habitats. Condor, 92, 4553.

Kusch, A., \& Henríquez, J. M.(2014). Respuesta del ensamblaje de aves passerines a la alteración del matorral por prácticas de manejo ganadero en Chile austral $\left(53^{\circ} \mathrm{S}\right)$. Revista Latinoamericana de Conservación, 4, 25-32.

Lazo, I., Anabalón, J. J., \& Segura, A. (1990). Perturbación humana del matorral y su efecto sobre un ensable de aves nidificantes de Chile central. Revista Chilena de Historia Natural, 63, 293-297.

MacArthur, R. H., \& MacArthur, J. M. (1961). On bird species diversity. Ecology, 42, 594598.

Martinic, M. (1985). La ocupación y el impacto del hombre sobre el territorio. In: O Boelcke, DM Moore \& FA Roig (Eds.), Transecta Botánica de la Patagonia Austral. Buenos Aires, Argentina.

Marticorena, A. (2006). Revision of the genus Acaena (Rosaceae) in Chile. Annals of the Missouri Botanical Garden, 93, 412-454.

Matthei, O. (1995).Manual de las malezas que crecen en Chile. Alfabeta Impresiones, Santiago de Chile.

McCune, B., \& Grace, J.B. (2002).Analysis of ecological communities. MJM Software Design. Oregon, USA.

McGehee, S., \& Eitniear, J. C. (2007).Diet of the Patagonian Sierra-Finch (Phrygilus patagonicus) on Navarino Island, Chile. Ornitología Neotropical, 18, 449-452. 
Milesi, F. A., Marone, L., Lopez de Casenave, J., Cueto, V. R., \& Mezquida, E. T. (2002). Gremios de manejo como indicadores de las condiciones del ambiente: un studio de caso con aves y perturbaciones del habitat en el Monte central, Argentina. Ecología Austral, 12, 149-161.

Moreno, C. E. (2001). Métodos para medir la biodiversidad. M\&T Manuales y Tesis SEA, vol. 1. Zaragoza.

Moore, D. M. (1983). Flora of Tierra del Fuego. Missouri Botanical Garden. Missouri, USA.

Motulsky, H. J. (2007).Prism version 5 Statistics Guide. GraphPad Software Inc., San Diego, USA.

Mueller-Dombois, D., \& Ellenberg, H.(1974).Aims and methods of vegetation ecology. John Wiley \& Sons Publishers. New York.

Muñoz-Pedreros, A., \& Merino, C. (2014).Diversity of aquatic bird species in a wetland complex in southern Chile. Journal of Natural History, 48, 1453-1465.

Noss, R. F. (1990). Indicators for monitoring biodiversity: A hierarchical approach. Conservation Biology, 4, 355-364.

Ojeda, V.,\& Trejo, A. (2002). First cavity-nesting records for three Patagonian forest birds. Hornero, 17, 85-89.

Petchey, O. L., \& Gaston, K. J. (2006). Functional Diversity: back to basics and looking forward. Ecology Letters, 9, 741-758.

Pisano, E. (1977). Fitogeografía de FuegoPatagonia chilena. I. Comunidades vegetales entre las latitudes $52^{\circ}$ y $56^{\circ} \mathrm{S}$. Anales del Instituto de la Patagonia, 8, 121-250.

R_Development_Core_Team (2012). R: a language and environment for statistical computing. Vienna, Austria. http: www.R-project.org.

Ralph, J. C. (1985). Habitat association patterns of forest and steppe birds of northern Patagonia, Argentina. The Condor, 97, 471-483

Ralph, J. C., Droege, S., \& Sauer, J. R. (1995). Managing and monitoring birds using point counts: standards and applications. USDA Forest Service Gen. Tech. Rep. PSWGTR-149.

Rau, J. R., Zuleta, C., Gantz, A., Saiz, F., Cortes, A., Yates, L., Spotorno, A. E., \& Couve,
E. (1998). Biodiversity of arthropods and terrestrial vertebrates of Chiles Norte Grande. Revista Chilena de Historia Natural, 71, 527-554.

Rodewald, A. D., \& Vitz, A. C. (2005). Edge- and area- sensitivity of shrubland birds. Journal of Wildlife Management, 69, 681-688.

Roberts, D. W. (2006). Labdsv: Laboratory for dynamic synthetic vegephenomenoloy. $\mathrm{R}$ package version 1.2-2. (http: cran.r-project. org).

Saavard, J. P. L., \& Hooper, T. D. (1997). Influence of survey length and radius size on grassland bird surveys by point counts at Williams Lake, British Columbia. En J. C. Ralph, J. R. Sauer \& S. Droege (Eds.), Monitoring bird populations by point counts. USDA Forest Service, General Technical Report PSWGTR-149, U. S. Department of Agriculture. Scherer, C., Jeltsch, F., Grimm, V., \& Blaum, N. (2016). Merging trait-based and individual base modeling: An animal functional type approach to explore the responses of birds to climatic and land use changes in semi-arid African savannas. Ecological Modelling, 326, 75-89.

Silva-Rodríguez, E. A., Ortega-Solis, G. R., \& Jiménez, J. E. (2008). Descripción del ensamble de aves en un agroecosistema del sur de Chile. Boletín Chileno de Ornitología, 14, 81-91.

Seymour, C. L., Simmons, R. E., Joseph, G. S., \& Slingsby, J. A. (2015) On bird functional diversity: species richness and functional differentiation show contrasting responses to rainfall and vegetation structure in an arid landscape. Ecosystems, 18, 971-984.

Sirami, C., Seymour, C., Midgley, G., \& Barnard, P. (2009). The impact of shrub encroachment on savanna bird diversity from local to regional scale. Diversity and Distributions, 15, 948-957.

Vander Haegen, W. M., Schroeder, M. A., \& DeGraaf, R. M. (2002). Predation on real and artificial nest in shrubsteppe landscapes fragmented by agriculture. Condor, 104, 496-506.

Vanderwalle, M., de Bello, F., Berg, M. P., Bolger, T., Dodelec, S., Dubs, F., Feld, C. K.,... 
Woodcock, B. A. (2010). Functional traits as indicators of biodiversity response to land use change across ecosystems and organisms. Biodiversity and Conservation, 19, 29212947.

Vickery, J. A., Tallowin, J. R., Feber, R. E., Asteraki, E. J., Atkinson, P. W., Fuller, R. J., \& Brown, V. K. (2001). The management of lowland neutral grasslands in Britain: effects of agricultural practices on birds and their food resources. Journal of Applied Ecology, 38, 647-664.

Vuilleumier, F. (1991). A quantitative survey of speciation phenomena in Patagonian birds. Ornitologia Neotropical, 2, 5-28.

Vuilleumier, F. (1995). Components of biodiversity in the avifauna of Patagonian steppes. Southern Connection Newsletter, 7, 6-17.

Vuilleumier, F. (1997). Status and distribution of Asthenes anthoides (Furnariidae), a species endemic to Fuego-Patagonia, with notes on its systematic relationships and conservation. Ornithological Monographs, 48, Studies in Neotropical Ornithology Honoring Ted Parker, 791-808.

Vuilleumier, F. (1998). Avian biodiversity in forest and steppe communities of Chilean fuego patagonia. Anales Instituto Patagonia, 26, 41-57.

Violle, C., Navas, M. L., Vile, D., Kazakou, E., Fortunel, C., Hummel, I., \& Garnier E. (2007). Let the concept of trait be functional! Oikos, 116, 882-892.

Wiens, J. A. (1989). The Ecology of Bird Communities, vol 1. Foundations and patterns. Cambridge University Press.

Wiens, J. A., \& Rotenberry, J. T. (1985).Response of breeding passerine birds to rangeland alterations in a North American shrubsteppe locality. Journal of Applied Ecology, 22, 655-668. 